\title{
Biaxial thin-film coated-plate polarizing beam splitters
}

\author{
lan Hodgkinson, Qi Hong Wu, Matthew Arnold, Lakshman De Silva, \\ Gisia Beydaghyan, Kate Kaminska, and Kevin Robbie
}

\begin{abstract}
We present a design for a biaxial thin-film coated-plate polarizing beam splitter that transmits the $p$-polarized component of a beam of light without change of direction and reflects the $s$-polarized component. The beam splitter has a periodic structure and is planned for fabrication by serial bideposition in mutually orthogonal planes. Recent experimental data for form-birefringent silicon is used to establish the feasibility of the design for a beam splitter to be used at $1310 \mathrm{~nm}$ and at an angle of $45^{\circ}$ in air. (C) 2006 Optical Society of America

OCIS codes: $160.1190,260.5430,260.1440,310.6860,310.1860,310.1210$.
\end{abstract}

\section{Introduction}

Many optical components and optical bench setups confine the propagation of light to a few selected directions, for example, along the length and width of an optical table. Elements such as polarizing-cube beam splitters ${ }^{1}$ are in common use but are relatively expensive optical components. In addition, prisms exhibit stress-related birefringence that changes with temperature and causes problems in imaging projectors. For these reasons there is currently a need in the optics community for a simple, coated-plate polarizing beam splitter that will operate in air $\left(n_{C}=1\right)$ at angles of incidence that include $\theta_{C}$ $=45^{\circ}$, as illustrated schematically in Fig. 1. However, the design of such an optical element is nontrivial when isotropic film materials are to be used. Basically, an impasse occurs because no pair of thinfilm materials satisfies the Brewster-angle condition ${ }^{2}$ for zero interfacial reflection of $p$-polarized light at $45^{\circ}$. This point is illustrated in Fig. 2, which plots combinations of refractive indices $n_{A}$ and $n_{B}$ that yield zero reflectance for $p$-polarized light incident at $45^{\circ}$ from a cover medium of refractive index $n_{C}$. The Brewster-index requirement, $n_{B}=n_{A}$ tan $\theta_{A}$, applies to the situation in which the reflection direction on

I. Hodgkinson (ijh@physics.otago.ac.nz), Q. H. Wu, M. Arnold, and L. De Silva are with the Department of Physics, University of Otago, P.O. Box 56, Dunedin, New Zealand. G. Beydaghyan, K. Kaminska, and K. Robbie are with the Department of Physics, Queen's University, Kingston, Ontario K7L 3N6, Canada.

Received 28 February 2005; revised 7 September 2005; accepted 25 October 2005.

0003-6935/06/071563-06\$15.00/0

(C) 2006 Optical Society of America one side of the interface and the direction of the refracted ray on the other side of the interface are perpendicular. In such a case no power is radiated in the reflection direction. Figure 2 implies that suitable thin-film materials may be selected to satisfy the Brewster condition when the refractive index $n_{C}$ of the cover medium is 1.5 , typical of a polarizing-cube beam splitter, but not when $n_{C}=1.0$ for a coated plate in air. Figure 2 also illustrates another situation that will yield zero reflectance of $p$-polarized light. Index matching, $n_{A}=n_{B}$, is trivial and not useful in stacks of isotropic materials, but it can be effective in stacks of birefringent materials. ${ }^{3-5}$

The purpose of this paper is to show that the $45^{\circ}$ coated-plate design is possible by using a pair of formbirefringent ${ }^{2,6}$ materials in a periodic structure. Such a stack of films can yield independent optical outputs for $p$-polarized and $s$-polarized light. ${ }^{7-9}$ Basically, we overcome the Brewster limitation by index matching the structure for $p$ polarization while leaving an independent and strongly reflecting structure for $s$ light. We begin by determining conditions that need to be satisfied by the principal refractive indices of the film materials, and then we use recent experimental data for biaxial silicon films ${ }^{10}$ to establish the feasibility of an all-silicon design. Finally we show that the planar thin-film polarizer for normally incident light, which has already been fabricated using alternating layers of form-birefringent titanium oxide and tantalum oxide, ${ }^{8}$ can be regarded as a special case of the polarizing beam splitter.

\section{Design Considerations}

Now we consider a periodic structure of two biaxial materials $\mathrm{A}$ and $\mathrm{B}$ with principal refractive indices $n_{\mathrm{A} 1}, n_{\mathrm{A} 2}, n_{\mathrm{A} 3}, n_{\mathrm{B} 1}, n_{\mathrm{B} 2}, n_{\mathrm{B} 3}$. Note that the subscripts 1 , 


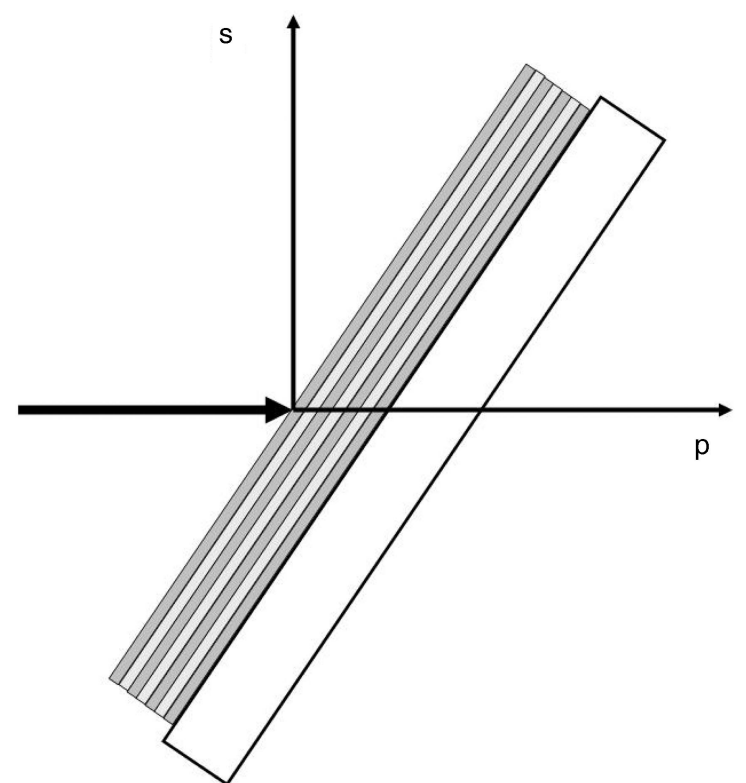

Fig. 1. Basic design of a thin-film coated-plate polarizing beam splitter.

2,3 that we use here to label the principal axes correspond to subscripts $z, x, y$ in some of our earlier publications. We assume that A and B are formed by serial bideposition ${ }^{10,11}$ at angles of vapor incidence of $\theta_{\mathrm{vA}}$ and $\theta_{\mathrm{vB}}$, as this leads to nanostructures that run perpendicular to the substrate. ${ }^{11,12}$ The refractive indices satisfy $n_{\mathrm{A} 1}>n_{\mathrm{A} 3}>n_{\mathrm{A} 2}, n_{\mathrm{B} 1}>n_{\mathrm{B} 3}>n_{\mathrm{B} 2}$, and with $\theta_{\mathrm{vA}}<\theta_{\mathrm{vB}}$ the indices of material $\mathrm{A}$ are larger than the corresponding indices of material $\mathrm{B}$.

As shown in Fig. 3, the principal axes A1 and B1 of the biaxial films are normal to the substrate and axes A2, A3; B2 and B3 are parallel to the substrate. The materials $\mathrm{A}$ and $\mathrm{B}$ are deposited in the mutually perpendicular planes A1 - A2 and B1 - B2. The plane of incidence for the oblique light is defined by axes $\mathrm{A} 1$ and $\mathrm{A} 2$, or equivalently by $\mathrm{B} 1$ and $\mathrm{B} 3$.

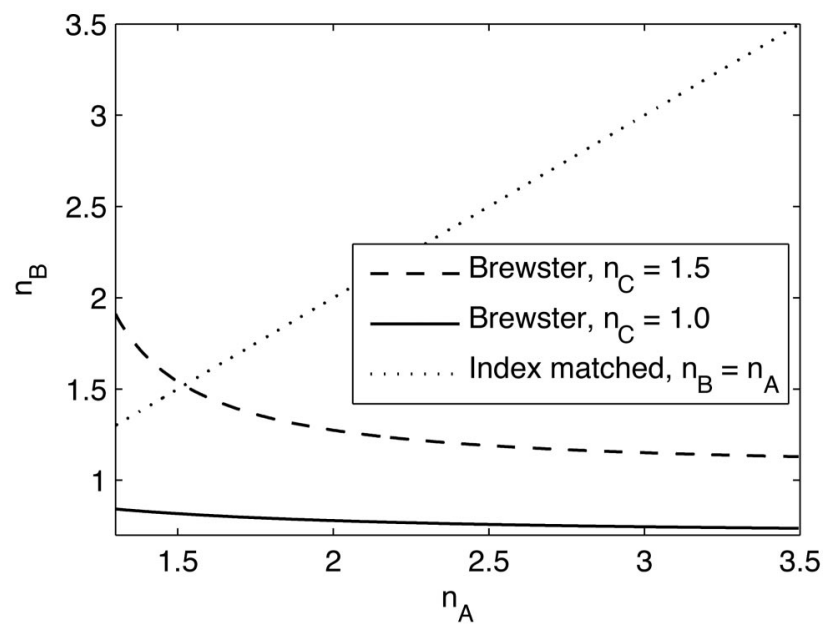

Fig. 2. Combinations of refractive indices $n_{\mathrm{A}}$ and $n_{\mathrm{B}}$ that yield zero reflectance for $p$-polarized light incident at $45^{\circ}$ from a cover medium of refractive index $n_{\mathrm{C}}$.

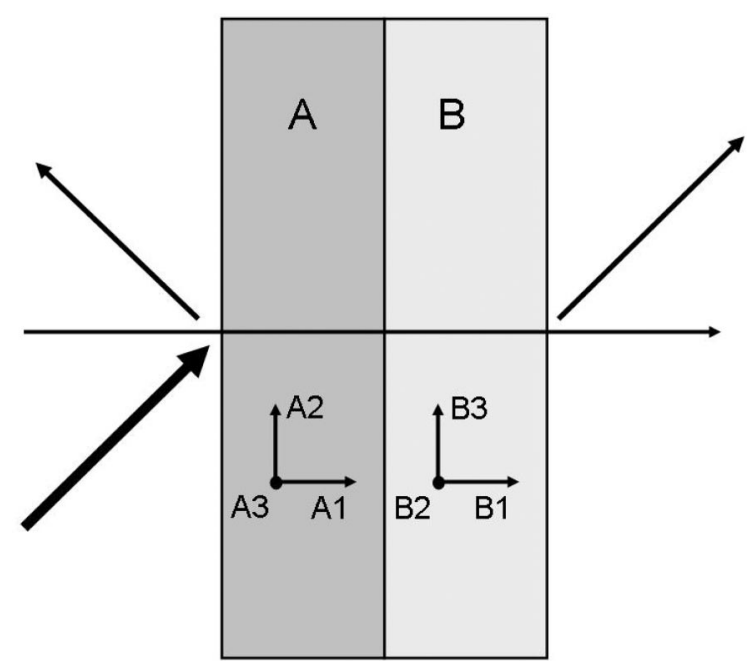

Fig. 3. One period of a biaxial thin-film stack for a coated-plate polarizing beam splitter, showing alignment of the principal axes in layers $\mathrm{A}$ and $\mathrm{B}$.

$P$-polarized or $s$-polarized light propagates in the plane of optical incidence without change of polarization, because the plane of incidence is a principal plane of both materials A and B.

Under these conditions, with zero cross-polarized reflectances and transmittances, computation can proceed via a pair of $2 \times 2$ field-transfer matrices, one for the $p$-polarization and the other for the $s$-polarization. Here we use equations that have been derived for these matrices and for the subsequent computation of the reflectances and transmittances of a multilayered stack of $N+1$ A layers and $N$ B layers. ${ }^{7}$

The condition for the interfaces of A and B to be nonreflecting for $p$ light to be incident obliquely can be expressed in terms of the field-ratio parameters

$$
\begin{aligned}
& \gamma_{\mathrm{A} p}=\left(1 / n_{\mathrm{A} 2}{ }^{2}-\beta^{2} / n_{\mathrm{A} 1}{ }^{2} n_{\mathrm{A} 2}{ }^{2}\right)^{-1 / 2}, \\
& \gamma_{\mathrm{B} p}=\left(1 / n_{\mathrm{B} 3}{ }^{2}-\beta^{2} /{n_{\mathrm{B} 1}}^{2} n_{\mathrm{B} 3}{ }^{2}\right)^{-1 / 2} .
\end{aligned}
$$

The amplitude reflection coefficient is

$$
r_{p}=\left(\gamma_{\mathrm{A} p}-\gamma_{\mathrm{B} p}\right) /\left(\gamma_{\mathrm{Ap} p}+\gamma_{\mathrm{B} p}\right),
$$

and hence we require $\gamma_{\mathrm{A} p}=\gamma_{\mathrm{B} p}$ for zero reflectance. Thus the condition can be expressed as

$$
n_{\mathrm{A} 1} n_{\mathrm{A} 2} /\left(n_{\mathrm{A} 1}^{2}-\beta^{2}\right)^{1 / 2}=n_{\mathrm{B} 1} n_{\mathrm{B} 3} /\left(n_{\mathrm{B} 1}^{2}-\beta^{2}\right)^{1 / 2} .
$$

The Snell's law invariant $\beta$ that appears in these equations can be determined by using the cover index $n_{C}$ and the angle of incidence $\theta_{C}$, thus $\beta=n_{C} \sin \theta_{C}$. In addition, we have omitted the parameter $z_{0}{ }^{7}$

An alternative but equivalent approach is to define effective refractive indices $n_{\mathrm{A} p}$ and $n_{\mathrm{B} p}$ for $p$ polarization as 


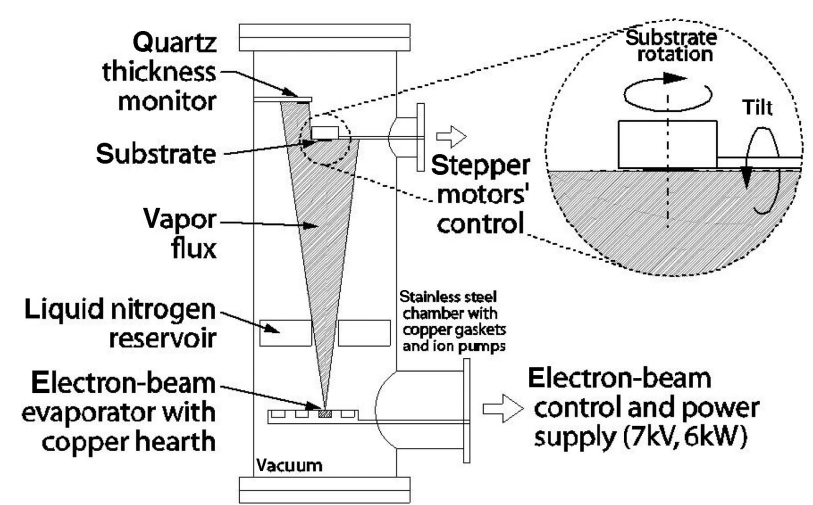

Fig. 4. Physical schematic of the evaporator used for depositing birefringent films.

$$
\begin{aligned}
& n_{\mathrm{A} p}=\left[\left(\gamma_{\mathrm{A} p}^{2}+\sqrt{\gamma_{\mathrm{A} p}^{4}-4 \gamma_{\mathrm{A} p}^{2} \beta^{2}}\right) / 2\right]^{1 / 2}, \\
& n_{\mathrm{B} p}=\left[\left(\gamma_{\mathrm{B} p}^{2}+\sqrt{\left.{\gamma_{\mathrm{B} p}^{4}-4 \gamma_{\mathrm{B} p}^{2} \beta^{2}}^{4}\right) / 2}\right]^{1 / 2} .\right.
\end{aligned}
$$

Then the condition for index matching for $p$-polarized light becomes

$$
n_{\mathrm{Ap}}=n_{\mathrm{B} p} \text {. }
$$

Next we consider the $s$ light at oblique incidence. In the A layers the $s$ light encounters the high refractive index of the reflecting stack, $n_{\mathrm{A} 3}$, and similarly the refractive index $n_{\mathrm{B} 2}$ of the $\mathrm{B}$ layers is the low index of the stack. For reflective resonance at wavelength $\lambda_{0}$ and at the oblique angle of incidence, the physical thicknesses of the layers (in Figs. 1 and 3) need to be

$$
\begin{aligned}
& d_{\mathrm{A}}=\lambda_{0} /\left[4\left(n_{\mathrm{A} 3}^{2}-\beta^{2}\right)^{1 / 2}\right], \\
& d_{\mathrm{B}}=\lambda_{0} /\left[4\left(n_{\mathrm{B} 2}^{2}-\beta^{2}\right)^{1 / 2}\right] .
\end{aligned}
$$

\section{Deposition of Form-Birefringent Silicon}

The birefringent silicon films were prepared by electron-beam evaporation by using the technique of glancing angle deposition (GLAD). Figure 4 shows an outline of the GLAD system at Queen's University. The substrate is situated on a manipulator stage, $50 \mathrm{~cm}$ directly above a $25 \mathrm{~mm}$ diameter crucible, in a hearth, where the silicon is melted and evaporated with a $7 \mathrm{kV}$ electron beam. A dry scroll pump, a loadlock turbomolecular pump, and two large ion pumps maintain the base pressure at $5 \times 10^{-9}$ Torr. A liquid nitrogen reservoir is kept filled throughout the deposition to provide chamber cooling and to counter a rise in background pressure from outgassing. The manipulator stage motion is managed with two computer-controlled stepper motors that tilt and rotate the substrate about its center. The stepper motors are controlled with a program written in the LabVIEW instrumentation development environment. The film thickness and deposition rate are measured throughout with a quartz-crystal thickness monitor, and the information is passed to the Lab-
VIEW control software. The GLAD system is described in more detail in Ref. 13.

The birefringent silicon films were deposited onto Corning 7059 glass substrates with serial bideposition at tilt angles of $20,40,50,60,70,75,80$, and 85 deg. In serial bideposition the substrate is tilted relative to the arriving vapor flux, and it is rotated about its normal with periodic film subdeposits made at azimuthal angles of $0,180,360, \ldots$ deg by means of pausing the substrate rotation and depositing a small fixed amount of vapor. ${ }^{11,14,15}$ All birefringent silicon films were manufactured with a nominal thickness of $200 \mathrm{~nm}$, as measured with the quartz-crystal thickness monitor situated near the substrate. The subdeposit thickness was kept constant at $5 \mathrm{~nm}$ for all films. The deposition rate was approximately $0.5 \mathrm{~nm} / \mathrm{s}$, and the time taken by the stepper motor to rotate the substrate through each 180 deg rotation was approximately $1-2 \mathrm{~s}$.

\section{Principal Refractive Indices of Biaxial Silicon}

Tables of the values of the principal refractive indices $n_{1}, n_{2}$, and $n_{3}$ of silicon deposited by using the apparatus described in Section 3 now form a primary resource for the investigation of biaxial coating designs. Separate tables for deposition angles $\theta_{v}$ of 20,40, 50, $60,70,75,80$, and 85 deg list the $n$ 's for 400 wavelengths $\lambda_{0}$ in the range 500 to $1500 \mathrm{~nm}$. In the current investigation, we use $\lambda_{0}=1310 \mathrm{~nm}$ and proceed to (i) extrapolate to this wavelength within the tables and (ii) fit quadratic expressions to the principal indices for deposition angles in the range 50 to $75 \mathrm{deg}$ :

$$
\begin{aligned}
& n_{1}=1.6531+4.2123 \theta_{v}-2.5252 \theta_{v}{ }^{2}, \\
& n_{2}=4.5239-1.8987 \theta_{v}-0.1660 \theta_{v}{ }^{2}, \\
& n_{3}=3.2871+0.9393 \theta_{v}-1.5155 \theta_{v}{ }^{2} .
\end{aligned}
$$

In Eqs. (10)-(12) the angle $\theta_{v}$ is to be entered in radian units, making them compatible with equations that were listed previously for obliquely deposited tantalum oxide, titanium oxide, and zirconium oxide. ${ }^{16}$

\section{Silicon Beam Splitters}

Figures 5 and 6 were generated by using Eqs. (10) (12) for form-birefringent silicon at $\lambda_{0}=1310 \mathrm{~nm}$. Figure 5 plots the principal refractive indices, and Fig. 6 shows the in-plane linear birefringence $n_{3}-n_{2}$. Both figures are annotated at a pair of deposition angles, $\theta_{\mathrm{vA}}=62^{\circ}$ and $\theta_{\mathrm{vB}}=68^{\circ}$, that provide index matching at $45^{\circ}$ and optimize the extinction ratio ( $p$ transmittance/s transmittance) of the plate polarizer. In the simulations, Eq. (4) is used as a test for index matching, but in practice (during fabrication) it would be unreasonable to expect accurate knowledge of the $n$ 's. Instead, the computed values of $\theta_{\mathrm{vA}}$ and $\theta_{\mathrm{vB}}$ are used as initial values, and test depositions are carried out to approach index matching iteratively. The calculated transmittances for a beam 


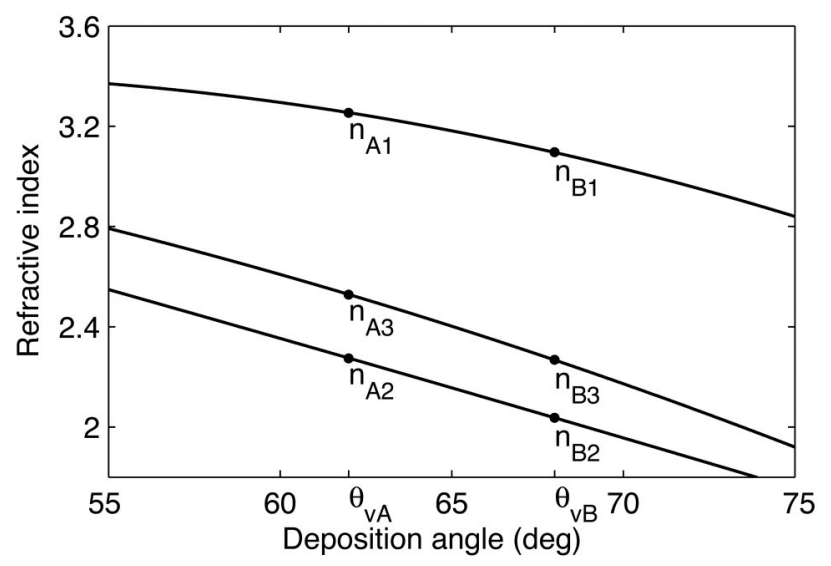

Fig. 5. Principal refractive indices of bideposited silicon.

splitter with cover index $n_{C}=1$, substrate index $n_{S}=1.52$, and $N=9$ (a total of 19 layers) are shown in Fig. 7. For these calculations we used the values appropriate to the marked points on Fig. 5: $n_{\mathrm{A} 1}=$ $3.254, n_{\mathrm{A} 2}=2.275, n_{\mathrm{A} 3}=2.529, n_{\mathrm{B} 1}=3.097, n_{\mathrm{B} 2}$ $=2.038$, and $n_{\mathrm{B} 3}=2.269$. The interference modulation on the $p$ transmittance can be mitigated by choosing an appropriate $N$ to move the maximum

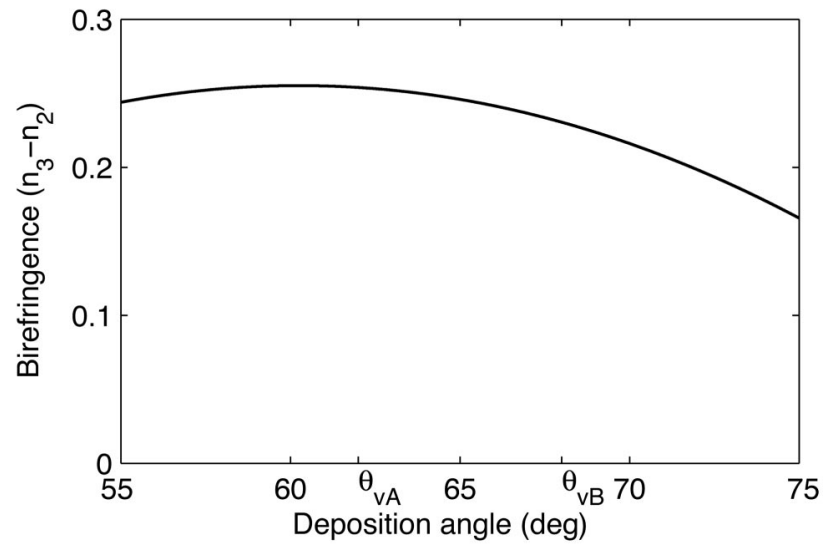

Fig. 6. Birefringence $n_{3}-n_{2}$ of bideposited silicon.

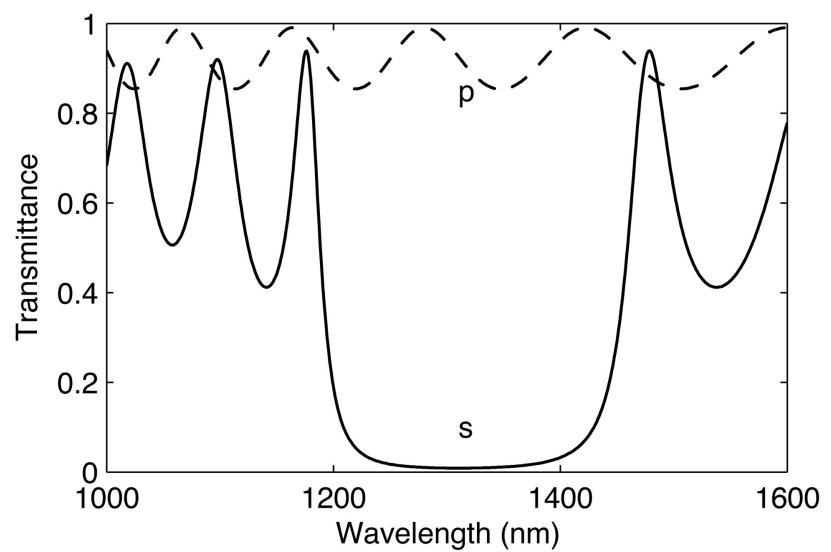

Fig. 7. Simulated transmittances of an all-silicon $45^{\circ}$ polarizing beam splitter.

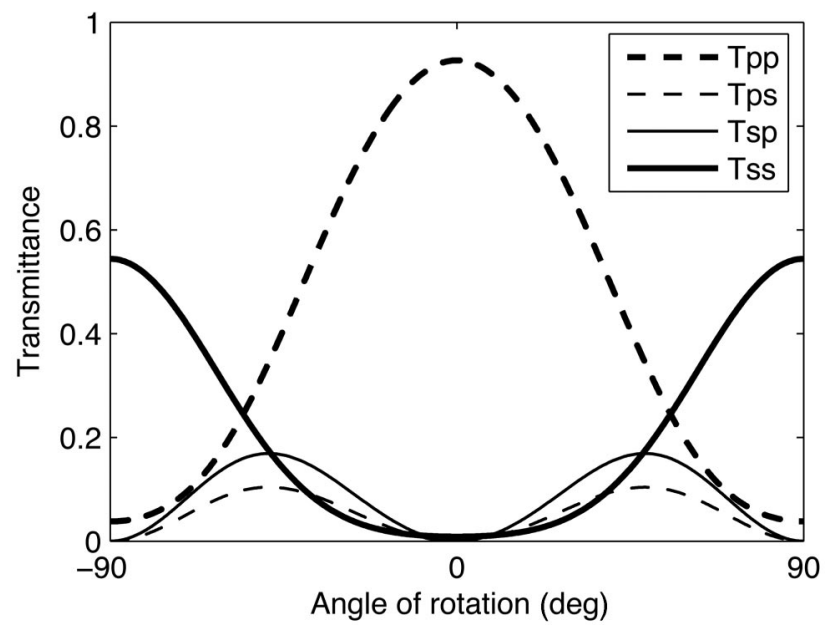

Fig. 8. Calculated dependence of copolarized and cross-polarized transmittances of the $45^{\circ}$ polarizing beam splitter on an azimuthal angle.

to the design wavelength. In addition, antireflection coatings are needed for the back of the substrate, particularly for a silicon plate. ${ }^{17}$

The beam splitter that is simulated in Fig. 7 has an extinction ratio $e=T_{p p} / T_{s s}>100$ when it is oriented according to the design angles. Ideally, the crosspolarized transmittances $T_{p s}$ and $T_{s p}$ are both zero under these conditions. However $4 \times 4$ matrix calculations ${ }^{7}$ show that misalignment of the beam splitter causes a change in performance. Thus Fig. 8. shows the effects of rotation about an azimuthal (normal) axis while a $45^{\circ}$ angle of optical incidence is maintained. Similarly, the extinction ratio depends on the optical angle of incidence, as shown in Fig. 9.

In general the extinction ratio of a given beam splitter tends to increase with increasing angle of incidence owing to larger $s$ reflections at the layer-tolayer interfaces and tends to decrease as the reflection band moves toward the blue end of the spectrum and away from the design wavelength. The first effect

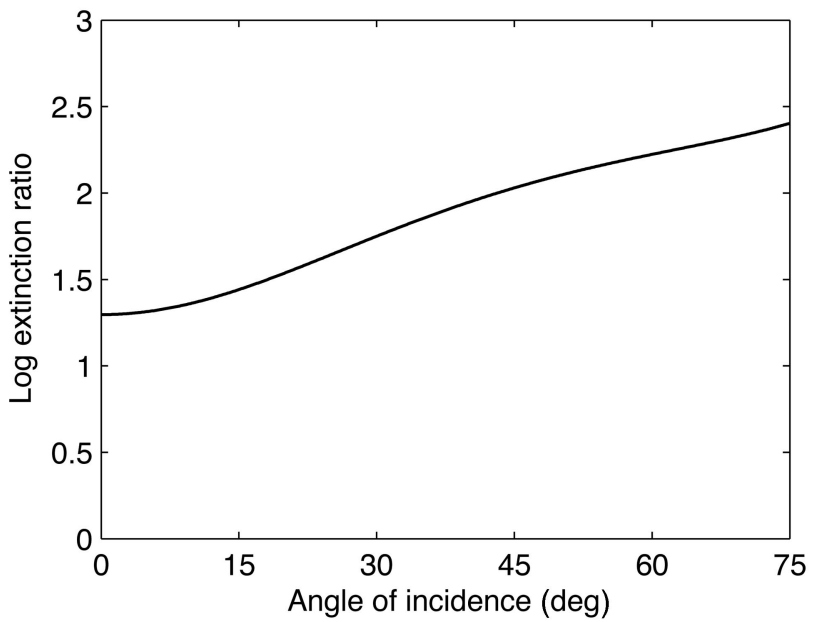

Fig. 9. Calculated dependence of the extinction ratio of the beam splitter on an optical angle of incidence. 


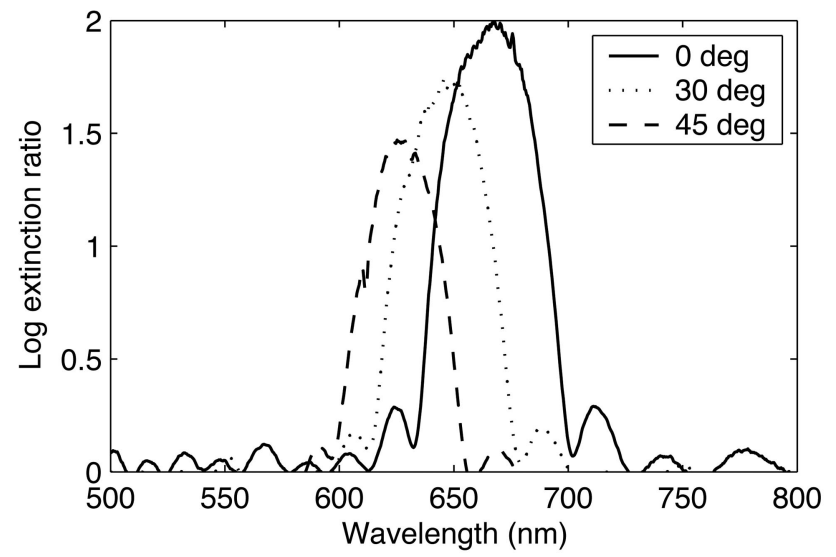

Fig. 10. Experimental curves showing the dependence of the extinction ratio of a titanium oxide- tantalum oxide polarizing beam splitter on an optical angle of incidence.

dominates in the case of the silicon beam splitter, which has a relatively broad bandwidth, but the second effect is prominent in systems such as titanium oxide. Experimental measurements (Fig. 10) made on a titanium oxide-tantalum oxide polarizing beam splitter $^{8}$ deposited for use at normal incidence show a reduction of $e$ with increasing $\theta$ but confirm maintenance of basic optical characteristics at larger angles of incidence.

Finally we note that further simulations indicate possible strategies for minimizing the dependence of the extinction ratio on the angle of incidence, such as the use of films that are quarter-waves at an angle smaller than the working angle of $45^{\circ}$.

\section{Discussion}

We have described the design of biaxial thin-film coated-plate polarizing beam splitters, focusing on elements for use at $45^{\circ}$ in air. The critical features of the design include (i) birefringent films with principal axes oriented perpendicular and parallel to the surface of the substrate to allow $p$-polarized and $s$-polarized light to propagate independently, (ii) index matching to provide high $p$ transmittance, and (iii) substantial high index/low index ratio to provide high $s$ reflectance. Recent experimental data recorded for formbirefringent silicon have been used in simulations to establish the feasibility of the basic design for nearinfrared (NIR) wavelengths. Other materials, fabrication methods, wavelengths, and optical angles of incidence can be used. Thus Tyan et al. ${ }^{18}$ have discussed a design based on a form-birefringent multilayer binary grating, Motohiro et al. ${ }^{19}$ have used simultaneous oblique deposition from two sources to grow anisotropic films with a normal axis, in-plane birefringence has been reported for spatially nanostructured silicon by Kovalev et al. ${ }^{20}$ and index matching has been achieved in multilayer polymer mirrors by Weber et al. ${ }^{9}$ Adaptation of our basic design to normal incidence leads to a planar thin-film polarizer, which has been fabricated for visible wavelengths by using alternating layers of form-birefringent titanium oxide and form-birefringent tantalum oxide. Potential development areas cover a wide wavelength range from NIR applications in telecommunications to planar, form-birefringent $\mathrm{LaF}_{3}$ polarizers for use at $193 \mathrm{~nm}$ and other deep-ultraviolet wavelengths.

The authors gratefully acknowledge the financial support received from the New Zealand Foundation for Research, Science and Technology; the MacDiarmid Institute for Advanced Materials and Nanotechnology (New Zealand); the Natural Sciences and Engineering Research Council of Canada; and the Canadian Institute for Photonic Innovations.

\section{References}

1. J. A. Dobrowolski, Handbook of Optics, M. Bass, ed. (McGrawHill, 1995), Vol. 1, Chap. 42.

2. M. Born and E. Wolf, Principles of Optics (Pergamon, 1959).

3. M. Elshazly-Zaghloul and R. M. Azzam, "Brewster and pseudoBrewster angles of uniaxial crystal surfaces and their use for determination of optical properties," J. Opt. Soc. Am. 72, 657661 (1982)

4. M. Elshazly-Zaghloul and R. M. Azzam, "Brewster and pseudoBrewster angles of uniaxial crystal surfaces and their use for determination of optical properties: errata,” J. Opt. Soc. Am. A 6, 607 (1989).

5. J. Lekner, "Brewster angles in reflection by uniaxial crystals," J. Opt Soc. Am. A 10, 2059-2064 (1993).

6. T. Motohiro and Y. Taga, "Thin film retardation plate by oblique deposition," Appl. Opt. 28, 2466-2482 (1989).

7. I. J. Hodgkinson and Q. H. Wu, Birefringent Thin Films and Polarizing Elements (World Scientific, 1998).

8. I. J. Hodgkinson and Q. H. Wu, "Birefringent thin-film polarizers for use at normal incidence and with planar technologies,” Appl. Phys. Lett. 74, 1794-1796 (1999).

9. M. F. Weber, C. A. Stover, L. R. Gilbert, T. J. Nevitt, and A. J. Ouderkirk, "Giant birefringent optics in multilayer polymer mirrors," Science 287, 2451-2456 (2000).

10. G. Beydaghyan, K. Kaminska, T. Brown, and K. Robbie, "Enhanced birefringence in vacuum evaporated silicon thin films," Appl. Opt. 43, 5343-5349 (2004).

11. I. J. Hodgkinson and Q. H. Wu, "Serial bideposition of anisotropic thin films with enhanced linear birefringence," Appl. Opt. 38, 3621-3625 (1999).

12. I. J. Hodgkinson, Q. H. Wu, M. J. Brett, and K. Robbie, "Vacuum deposition of biaxial films with surface-aligned principal axes and large birefringence $\Delta n$," in Optical Interference Coatings, Vol. 9 of 1998 OSA Technical Digest Series (Optical Society of America, 1998), pp. 104-106.

13. K. Robbie, G. Beydaghyan, T. Brown, C. Dean, J. Adams, and C. Buzea, "Ultrahigh vacuum glancing angle deposition system for thin films with controlled 3-D nanoscale structure," Rev. Sci. Instrum. 75, 1089-1097 (2004).

14. M. Suzuki and Y. Taga, "Anisotropy in the optical absorption of $\mathrm{Ag}-\mathrm{SiO}_{2}$ thin films with oblique columnar structures," J. Appl. Phys. 71, 2448-2454 (1992).

15. K. Robbie, L. Friedrich, S. Dew, T. Smy, and M. Brett, "Fabrication of thin films with highly porous microstructures," J. Vac. Sci. Technol. A 13, 1032-1035 (1995).

16. I. J. Hodgkinson, Q. H. Wu, and J. C. Hazel, "Empirical equations for the principal refractive indices and column angle of obliquely deposited films of tantalum oxide, titanium oxide and zirconium oxide," Appl. Opt. 37, 2653-2659 (1998).

17. Q. H. Wu, L. De Silva, M. D. Arnold, I. J. Hodgkinson, and E. Takeuchi, "All-silicon polarizing filters for near infrared wavelengths,” J. Appl. Phys. 95, 402-404 (2004). 
18. R.-C. Tyan, P.-C. Sun, A. Scherer, and Y. Fainman, "Polarizing beam splitter based on the anistropic spectral reflectivity characteristic of form-birefringent multilayer gratings," Opt. Lett. 21, 761-763 (1996).

19. T. Motohiro, Y. Takeda, T. Hioki, and S. Noda, "Simultaneous oblique deposition from opposite azimuthal directions for fabrication of thin film retardation plates," in International Sym- posium on Polarization Analysis and Applications to Device Technology, T. Yoshizawa and H. Yokota, eds., Proc. SPIE 2873, 214-217 (1996).

20. D. Kovalev, G. Polisski, J. Diener, H. Heckler, N. Künzner, V. Yu. Timoshenko, and F. Koch, "Strong in-plane birefringence of spatially nanostructured silicon," Appl. Phys. Lett. 78, 916918 (2001). 